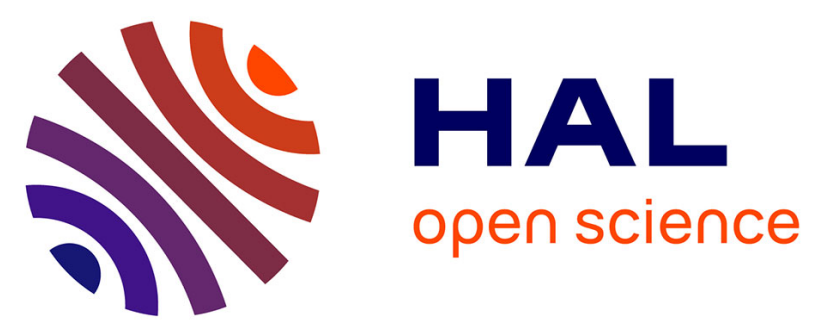

\title{
Generation of nonlinear electric field bursts in the outer radiation belt through the parametric decay of whistler waves
}

O. V. Agapitov, V Krasnoselskikh, F. S. Mozer, A.V. Artemyev, A.S. Volokitin

\section{- To cite this version:}

O. V. Agapitov, V Krasnoselskikh, F. S. Mozer, A.V. Artemyev, A.S. Volokitin. Generation of nonlinear electric field bursts in the outer radiation belt through the parametric decay of whistler waves. Geophysical Research Letters, 2015, 42, pp.3715-3722. 10.1002/2015GL064145 . insu-01174881

\author{
HAL Id: insu-01174881 \\ https://hal-insu.archives-ouvertes.fr/insu-01174881
}

Submitted on 10 Jul 2015

HAL is a multi-disciplinary open access archive for the deposit and dissemination of scientific research documents, whether they are published or not. The documents may come from teaching and research institutions in France or abroad, or from public or private research centers.
L'archive ouverte pluridisciplinaire HAL, est destinée au dépôt et à la diffusion de documents scientifiques de niveau recherche, publiés ou non, émanant des établissements d'enseignement et de recherche français ou étrangers, des laboratoires publics ou privés. 


\section{Geophysical Research Letters}

\section{RESEARCH LETTER \\ 10.1002/2015GL064145}

\section{Key Points:}

- Whistler wave parametric decay is observed and studied

- The conditions of electron acoustic wave weak damping in are found

- The source of nonlinear electric field bursts (TDS) is found

Supporting Information:

- Readme

- Text S1 and Figure S1

Correspondence to:

O. V. Agapitov,

oleksiy.agapitov@gmail.com

\section{Citation:}

Agapitov, O. V., V. Krasnoselskikh, F. S. Mozer, A. V. Artemyev, and A. S. Volokitin (2015), Generation of nonlinear electric field bursts in the outer radiation belt through the parametric decay of whistler waves, Geophys. Res. Lett., 42, 3715-3722, doi:10.1002/2015GL064145.

\section{Received 6 APR 2015}

Accepted 29 APR 2015

Accepted article online 5 MAY 2015

Published online 27 May 2015
O2015. American Geophysical Union. All Rights Reserved.

\section{Generation of nonlinear electric field bursts in the outer radiation belt through the parametric decay of whistler waves}

\author{
O. V. Agapitov ${ }^{1,2}$, V. Krasnoselskikh ${ }^{1,3}$, F. S. Mozer ${ }^{1}$, A. V. Artemyev ${ }^{4}$, and A. S. Volokitin ${ }^{4}$ \\ ${ }^{1}$ Space Sciences Laboratory, University of California, Berkeley, California, USA, ${ }^{2}$ Astronomy and Space Physics Department, \\ Taras Shevchenko National University of Kiev, Kiev, Ukraine, ${ }^{3}$ LPC2E/CNRS, University of Orleans, Orleans, France, ${ }^{4}$ Space \\ Research Institute, Russian Academy of Sciences, Moscow, Russia
}

Abstract Huge numbers of different nonlinear structures (double layers, electron holes, nonlinear whistlers, etc., referred to as Time Domain Structures, TDS) have been observed by the electric field experiment on the Van Allen Probes. Some of them are associated with whistler waves. Such TDS often emerge on the forward edges of the whistler wave packets and form chains. The parametric decay of a whistler wave into a whistler wave propagating in the opposite direction and an electron acoustic wave is studied experimentally as well as analytically, using Van Allen Probes data. The resulting electron acoustic wave is considered to be the source of electron scale TDS. The measured parameters of the three waves (two whistlers and the electron acoustic wave) are in good agreement with an assumption of their parametric interaction: $\omega_{0}=\omega_{1}+\omega_{2}$ and $\vec{k}_{0}=\vec{k}_{1}+\vec{k}_{2}$. The bicoherence analysis shows the nonlinear nature of the observed electron-acoustic waves as well as the whistler wave and electron acoustic wave phase relation. The estimated decay instability growth rate shows that the process of three-wave interaction can develop in a characteristic time smaller than $1 \mathrm{~s}$, thus, the process is rapid enough to explain the observations. This induced parametric interaction can be one of the mechanisms for quasiperiodic TDS generation in the outer Van Allen radiation belt.

\section{Introduction}

Huge numbers of short duration electric field spikes, called Time Domain Structures (TDS), are observed by Van Allen Probes spacecraft in the outer radiation belts [Mozer et al., 2013]. Regular observations of such nonlinear structures in association with injections or boundaries in the night sector of the magnetosphere [Malaspina et al., 2014] make them a frequently observed feature of the radiation belt environment. TDS can efficiently interact with thermal electrons, accelerating them to several keV energies and scattering them into the loss cone through Landau resonance trapping [Artemyev et al., 2014]. Thus, TDS can play a role in locally producing the nonthermal population of 1-10 keV and electron precipitation [Mozer et al., 2014]. Observed TDS were identified as nonlinear electron acoustic mode waves because of their temporal and spatial scales. The multiple sources of these waves in the outer radiation belts remain unclear. In laboratory laser plasma experiments, such waves are often generated by the parametric instability of electromagnetic waves [see, for example, Montgomery et al., 2001, and references therein]. The parametric instability in a plasma is a nonlinear interaction in which a pump wave at frequency $\omega_{0}$ is converted into two waves at frequencies $\omega_{1}$ and $\omega_{2}$ with

$$
\omega_{0}=\omega_{1}+\omega_{2}, \vec{k}_{0}=\vec{k}_{1}+\vec{k}_{2}
$$

where $\vec{k}_{0}, \vec{k}_{1}$, and $\vec{k}_{2}$ are the wave vectors of corresponding waves. In laser plasma research, intense laser-emitted electromagnetic wave can couple to weakly damped electrostatic waves in the plasma and produce scattered light waves. Electron acoustic waves (EAWs) have large linear damping rates in Maxwellian plasma distributions [Stix, 1962; Fried and Gould, 1961; Montgomery et al., 2001]. However, it was found [Schamel, 2000; Holloway and Dorning, 1991, and references therein] that, in nonlinear Vlasov-Maxwell systems, electrons trapped in the wave electrostatic potential can result in undamped solutions [Bernstein et al., 1957], thereby allowing the EAW to exist in a broad range of laboratory laser plasma conditions [Nikolic et al., 2002; Montgomery et al., 2001]. 
The dynamics of electrons of the outer radiation belt is thought to be controlled mainly by chorus-type whistler waves [Thorne, 2010] which are structured electromagnetic emissions observed in two frequency ranges: lower band chorus in the range from 0.1 to 0.5 of the local electron gyrofrequency, $f_{\text {ce, }}$, and upper band chorus in the frequency range from 0.5 to $f_{\text {ce. }}$. It has been shown [Galeev and Sagdeev, 1973] that an electron whistler wave propagating along the background magnetic field can decay into a secondary whistler wave and a lower frequency ion acoustic wave [Shukla, 1975] or Alfven wave [Chen, 1977], depending on plasma parameters. The possibility of the decay of a whistler wave into EAW has not been considered before because of its supposed large linear damping rate. In this paper we show the first experimental evidence that the generation of quasiperiodic electron acoustic TDS in the Earth's outer radiation belt can result from the parametric decay of a primary whistler wave into a secondary whistler wave (with shifted frequency and propagating in the opposite direction) and a low-frequency, weakly damped EAW that evolves, via efficient trapping of electrons, into electric field bursts.

\section{Observations}

Van Allen Probes A and B were launched on 30 August 2012. Electric and magnetic field waveforms were produced by the Electric Fields and Waves (EFW) [Wygant et al., 2013] and the Electric and Magnetic Field Instrument Suite and Integrated Science [Kletzing et al., 2013] detectors. These data were transmitted at 16,384 samples/s for $5 \mathrm{~s}$ intervals in a burst mode. The burst mode is triggered by large-amplitude signal for $5 \mathrm{~s}$ recording or started manually for arbitrary time interval. Here we present the analysis of such waveform data captured by Van Allen Probes B on 1 November 2012 when about 14 intervals (of $5 \mathrm{~s}$ burst measurements) were collected during 08:12-08:14 UT. Van Allen Probes B during this time interval was located in the morning sector (MLT $=5.4$ ) at $L=5.5$ in the vicinity of the geomagnetic equator (magnetic latitude, $\lambda$, was about $2^{\circ}$ ). Plasmapause crossings were detected at $L \sim 4.1$ (outbound crossing at 06:30) and at $L \sim 3.5$ (inbound crossing at 12:50). This interval has previously been studied by Mozer et al. [2013] to ascertain properties and durations of TDS. During this interval, the ambient magnetic field, of magnitude $\sim 125 \mathrm{nT}$, was within $10^{\circ}$ of the satellite spin plane (the plane approximately normal to the Sun-Earth line) and the spin plane contained electric field measuring spheres 1, 2, 3, and 4, each at the end of a $50 \mathrm{~m}$ wire, while shorter booms with spheres on their ends were located parallel to the spin axis [Wygant et al., 2013]. This allowed estimation of the propagation direction (equatorward along the magnetic field line) and velocity of the TDS. They were observed in the presence of intensive (about $50 \mathrm{pT}$ in magnetic field and about $5 \mathrm{mV} / \mathrm{m}$ in electric field) whistler waves. The $\sim 1 \mathrm{~ms}$ bursts of electric field appeared in localized groups of spikes with typical durations of about $1 \mathrm{~s}$, with each group containing hundreds of spikes. One of the groups is shown in Figure 1.

Figure 1 illustrates the three fluctuating components of the electric and magnetic fields in backgroundmagnetic-field-aligned coordinates during a $1.5 \mathrm{~s}$ interval on 1 November 2012 (see also Mozer et al. [2013] for additional information). Figures $1 \mathrm{a}-1 \mathrm{c}$ and $1 \mathrm{e}-1 \mathrm{~g}$ give the components of the magnetic and electric field respectively with Figures $1 \mathrm{c}$ and $1 \mathrm{~g}$ giving the components parallel to the magnetic field. A series of electric field spikes is observed in the parallel electric field component. Figures $1 \mathrm{~d}$ and $1 \mathrm{~h}$ give the dynamic spectra of the perpendicular magnetic field perturbation and the parallel electric field component. The magnetic field dynamic spectrum shows that the whistler waves had two well-separated frequency maxima at $\sim 1.5-1.6 \mathrm{kHz}$ (below $0.5 f_{\text {ce }}$ ) and at $\sim 1.8-1.9 \mathrm{kHz}$ (above $0.5 f_{\text {ce }}$ ). Figure $1 \mathrm{i}$ shows the detailed structure of the parallel electric field during a portion of the time interval shown in Figures $1 \mathrm{a}-1 \mathrm{~h}$. The structure of electric field spikes from a later time interval is shown in Figure $1 \mathrm{j}$. Figures $1 \mathrm{i}$ and $1 \mathrm{j}$ present different stages of the nonlinear structure development with Figure 1 i showing electrostatic, partially steepened, sawtooth-like structures having positive and negative perturbations, and Figure $1 \mathrm{j}$ showing solitary fully formed double layers (this interval is from an earlier group of TDS that may have been formed a larger distance from the observation point).

The more detailed waveforms of the $X$ component of the perpendicular magnetic field and the parallel electric field for a $200 \mathrm{~ms}$ interval are presented in Figures $2 \mathrm{a}$ and $2 \mathrm{~b}$. The two whistler waves produce the low-frequency envelope of the whistler waveform seen in Figure 2a. The spectrum of the electric field spikes is seen in Figure $2 \mathrm{c}$ as the red curve covering the frequency range of $10-1000 \mathrm{~Hz}$. In this frequency range, the ratio of the magnetic and electric field amplitudes presented in Figures $2 \mathrm{~d}$ and $1 \mathrm{~h}\left(|\mathbf{B}| \sim 0.1 \mathrm{pT}^{2} / \mathrm{Hz}\right.$ and $|\mathbf{E}| \sim 0.1-1 \mathrm{mV}^{2} / \mathrm{m}^{2} \mathrm{~Hz}$ ) suggests that these are electrostatic structures. The low-frequency spectrum of Figure 2c shows that the noise-like signal was structured with a number of peaks. As seen in Figure $1 \mathrm{~g}$, the amplitude of the electric field perturbation varied from several to $25 \mathrm{mV} / \mathrm{m}$. From the analysis of the signal 


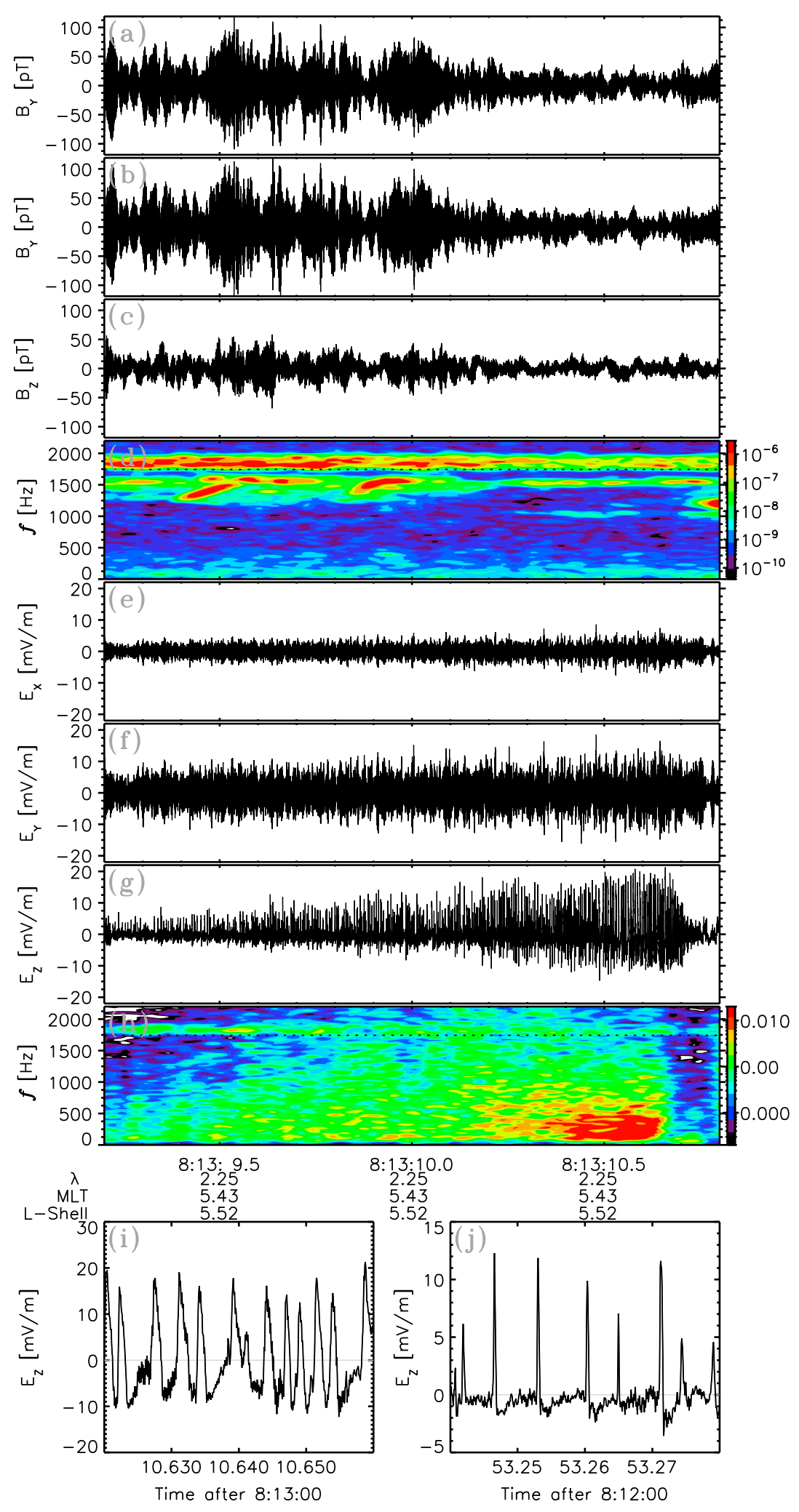

Figure 1. Three components of the $(a-c)$ magnetic and $(e-g)$ electric field perturbations in magnetic-field-aligned coordinates measured on Van Allen Probes B on 1 November 2012. Dynamic spectra of the (d) perpendicular magnetic and (h) parallel electric fields are shown (black dot lines indicate $0.5 f_{\mathrm{ce}}$ ). (i and j) More detailed $E_{z}$ waveforms. 

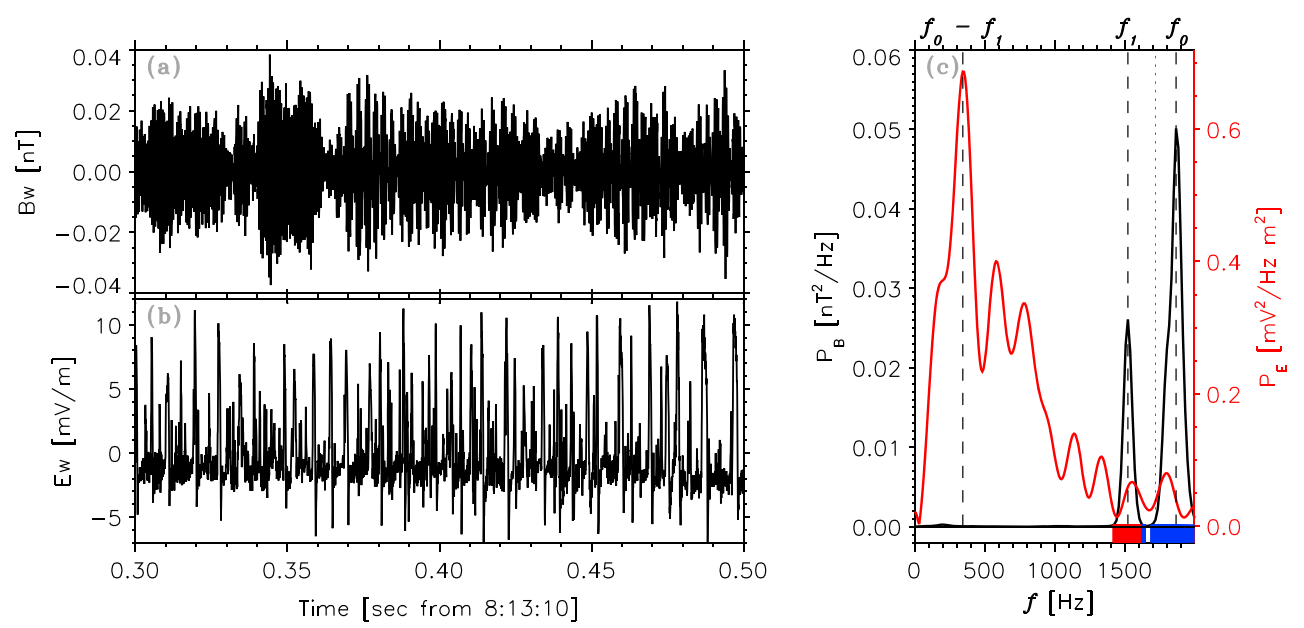

Figure 2. Dynamics of the (a) perpendicular $X$ component of the magnetic field and the (b) parallel $Z$ component of the electric field. (c) Spectra of the magnetic (black curve) and electric (red curve with the scale in the right) field perturbations during the time interval presented in Figures $2 \mathrm{a}$ and $2 \mathrm{~b}$. The Poynting flux direction is indicated in the bottom of Figure $2 \mathrm{c}$ with red being along the background magnetic field and blue in the opposite direction. The expression $0.5 f_{\text {ce }}$ is indicated by the dotted line

on the different electric field antennas [see Mozer et al., 2013; Artemyev et al., 2014 for details] the propagation velocity of the electrostatic structures was found to be about $2000-4000 \mathrm{~km} / \mathrm{s}$ (averaged value was $V_{\text {TDS }}=3100 \mathrm{~km} / \mathrm{s}$ ) and directed toward the geomagnetic equator. The whistler wavelengths are estimated from the approximate dispersion relation [Agapitov et al., 2013a] to be in the range from 21 to $22 \mathrm{~km}$ for the upper band and from 23.5 to $25 \mathrm{~km}$ for the lower band chorus.

The Poynting flux direction, shown as the color bars in the bottom of Figure. $2 \mathrm{c}$ with red being along the background magnetic field and blue being in the opposite direction, indicates the opposite directions of propagation of the lower and upper band chorus emissions. In this particular case the lower band whistlers were generated from the upper band whistler wave through the parametric decay process. However, this generation process likely cannot be considered as the regular one because the majority of upper and lower frequency band whistlers (chorus type) propagate in the same direction from the geomagnetic equator [Agapitov et al., 2013b]. Difference in speed of the upper and lower band waves for the processing case gives a range for the electron acoustic wave phase velocity from 2200 to $4500 \mathrm{~km} / \mathrm{s}$ (from $\frac{\omega_{0}-\omega_{1}}{k_{0}+k_{1}}$ ), close to the observed values. In the late nonlinear stage, the parallel spatial scale of a single spike in Figure $1 \mathrm{j}$ is about $0.5-1.0 \mathrm{~km}$, and the distance between them varies over the range from $10 \mathrm{~km}$ to $20 \mathrm{~km}$ (with an average of $11.5 \mathrm{~km}$ ). The observation of nonlinear electrostatic TDS provides an additional indication that the waves were generated by the instability associated with a whistler wave decaying to a secondary whistler wave and an electrostatic wave of the acoustic compressible type. The natural candidate for the acoustic wave might be an ion acoustic wave (IAW). But the observed TDS velocity $V_{\text {TDS }}$ was much larger than the ion sound velocity, so the waves presumably should belong to the EAW mode. EAW exists in plasmas with two or more electron populations, hot and cold, as was observed during the processed time interval, and their frequency is determined by the following relation [Stix, 1962]:

$$
\omega_{\mathrm{EAW}}^{2}=\frac{k_{z}^{2} C_{\mathrm{EAW}}^{2}}{1+k^{2} \lambda_{\mathrm{eH}}^{2}},
$$

here $C_{\mathrm{EAW}}^{2}=\frac{k_{\mathrm{B}} T_{\mathrm{eH}}}{m_{\mathrm{e}}} \frac{n_{\mathrm{eC}}}{n_{\mathrm{eH}}}=\lambda_{\mathrm{eH}}^{2} \omega_{\mathrm{peC}}^{2}$, where $n_{\mathrm{eH}}, n_{\mathrm{eC}}$ are hot and cold electron plasma densities, $k_{\mathrm{z}}$ is the magneticfield-aligned component of the $\vec{k}$ vector of the wave, $T_{\mathrm{eH}}$ is the temperature of the hot electron population, $\lambda_{\mathrm{eH}}=\left(k_{B} T_{\mathrm{eH}} / 4 \pi n_{\mathrm{eH}} e^{2}\right)^{1 / 2}$, is the Debye length of the hot electron population, and $\omega_{\mathrm{peC}}=\left(4 \pi n_{\mathrm{eH}} e^{2} / m_{e}\right)^{1 / 2}$, is the plasma frequency of the cold electron population. For this mode to be weakly damped its phase velocity must be larger than the thermal velocity of the cold electrons and smaller than the thermal velocity of hot electrons that can be satisfied if the density of the hot electron population is much larger than the density of the cold electron component. This condition may be relaxed if hot and cold populations move with respect to each other along the background magnetic field. In the observed case the hot component flows at velocity $V_{z}$ 
in the direction opposite to the background magnetic field (see Figure S1a of supporting information). In this case the dispersion relation in the reference frame of the hot (moving) component can be written as follows:

$$
\left(1+\frac{1}{k^{2} \lambda_{\mathrm{eH}}^{2}}\right)-\frac{\omega_{\mathrm{pec}}^{2}}{\left(\omega-k V_{z}\right)^{2}}=0
$$

taking into account that $k_{z}=k$. Then the solution reads

$$
\omega=k_{z} V_{z} \pm \omega_{\mathrm{peC}} \sqrt{\frac{k_{z}^{2} \lambda_{\mathrm{eH}}^{2}}{1+k_{\mathrm{z}}^{2} \lambda_{\mathrm{eH}}^{2}}}=k_{z} V_{z} \pm k_{z} C_{\mathrm{EAW}} \sqrt{\frac{1}{1+k_{z}^{2} \lambda_{\mathrm{eH}}^{2}}}
$$

If the hot plasma flow velocity $V_{z} \approx C_{\text {EAW }}\left(V_{z}\right.$ is estimated to be about $3000 \mathrm{~km} / \mathrm{s}$ which is close to the observed and estimated value of $V_{\text {TDS }}$ in the spacecraft reference frame) then EAW phase velocity in the flow frame is much less than the hot population thermal velocity $V_{\text {phEAW }}($ hot $)=V_{\text {TDS }}-V_{z}<v_{\text {TeH }}$ which makes EAW weakly damped, even when the density of cold plasma is greater than the density of hot plasma $n_{\mathrm{eco}}>n_{\mathrm{eHO}}$. The characteristic spatial scale when the growth of gradients or nonlinear steepening stops is determined by the effects related to the wave dispersion: the competition of nonlinear effects related to the wave amplitude and dispersive effects related to gradients of wave field [e.g., Whitham, 1974]. In the present case, it is determined by the characteristic scale $\lambda_{\mathrm{eH}}$, which can be estimated as $\sim 50-100 \mathrm{~m}$ by taking into account the measured values of $\omega_{\mathrm{eC}}, \omega_{\mathrm{EAW}}$, and $k$ of the EAW. The characteristic temperature of the hot electrons (obtained from fitting the distribution function provided by HOPE, see Figure $\mathrm{S} 1 \mathrm{~b}$ in the supporting information) is the order of $0.1-0.3 \mathrm{keV}$, corresponding to the trapped hot plasma density presumed to be about $1-3 \mathrm{~cm}^{-3}$ (from the obtained values of $\lambda_{\mathrm{eH}}$ ). The obtained density of trapped electrons is in good agreement with the results of PIC numerical simulations presented in Drake et al. [2015] and analytical estimations provided in Vasko et al. [2015], where the density of trapped particles was found to be close to the density of crossing ones. The same order as the cold plasma density $5-6 \mathrm{~cm}-3$ provided by HOPE ion moments. During the time interval of interest, the spacecraft was charged to $-70 \mathrm{~V}$ by the large flux of plasma electrons. Thus, electrons with energies below $70 \mathrm{eV}$ could not reach the detector and it is not possible to measure the main cold electron population. The same effect provided additional acceleration for thermal ions to be detected by HOPE. Because of this, electron moments cannot be used directly but should be corrected before on the value of charging. The average estimated density of the hot component from HOPE was $\sim 0.2-0.5 \mathrm{~s} \mathrm{~m}^{-3}$, an order of magnitude less than the cold plasma density, which is in good agreement with our estimations based on the $\lambda_{\mathrm{eH}}$ value.

The characteristic time for the development of the decay instability should be short as compared to the wave packet duration. This characteristic time can be evaluated by making use of the characteristic amplitude and wave characteristics of the whistler waves as follows: [Galeev and Sagdeev, 1973]:

$$
\tau^{-1} \sim \Gamma \sim\left[\frac{\omega_{2} k_{0} V_{\mathrm{gr}}\left(\omega_{1}, k_{1}\right)}{16 \pi n_{\mathrm{eH}} T_{\mathrm{eH}}}\left(\frac{c k_{0} E_{0}}{\omega_{0}}\right)^{2}\right]^{1 / 2} \sim 10 \mathrm{~s}^{-1} .
$$

where $V_{\mathrm{gr}}\left(\omega_{1}, k_{1}\right)$ is the group velocity of the low band chorus whistler wave estimated from the dispersion relation [Helliwell, 1965] and $E_{0}$ is the electric field of primary whistler wave. Taking electric field to be equal to $E_{0} \sim 1 \mathrm{mV} / \mathrm{m}$ the refraction index $N \sim 10(N=\mathrm{kc} / \omega)$, one can find $\Gamma \sim 10 \mathrm{~s}^{-1}$. Thus, the decay instability growth time is smaller than $1 \mathrm{~s}$, signifying that the process is rapid enough to explain the observations. It is worth noting that a similar process can occur when the third wave involved in the wave-wave interaction is the IAW. The dynamics of the IAW is similar to that described here for the EAW, the difference being the parameter range. The characteristic frequency of the lower wave mode in this case would be less than the ion plasma frequency, and the electron sound velocity in our analysis would be replaced by the ion sound speed. However, the major characteristics of the process would be the same, including formation of quasiperiodic chains, steepening and formation of the electric field bursts on the dispersion time scale, the distance between bursts determined by the wavelength of the decaying waves, and the electric field potential amplitude determined as a fraction of the thermal plasma energy. This IAW generation process has been studied in detail and manifestations of the described characteristics were evidenced by Sato and Okuda [1981].

The EAWs evolve into electric field bursts by steepening on the forward edges of the wave structures and finally form quasiperiodic chains of TDS. This dynamic behavior is similar to observations of compressible 


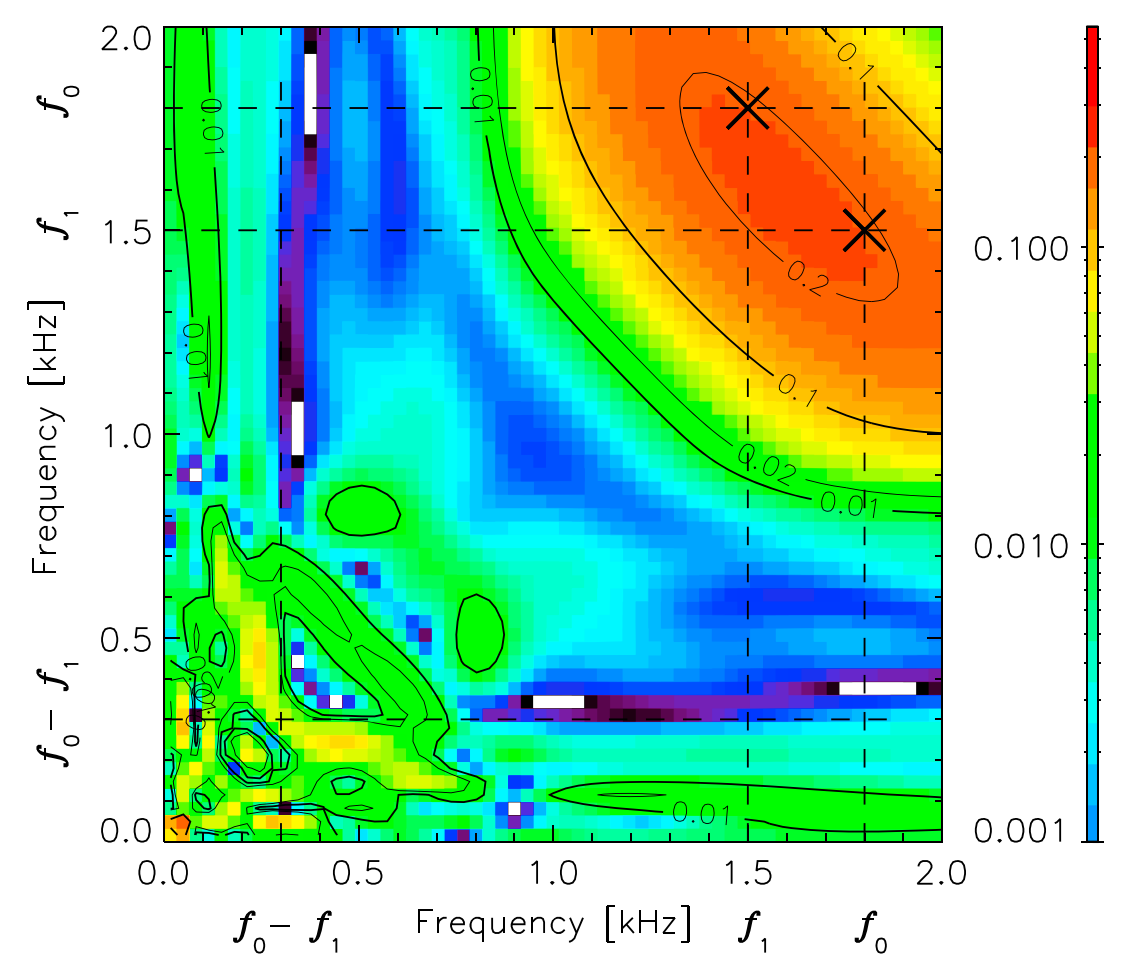

Figure 3. The bicoherence spectrum of the two transverse magnetic field components and the parallel component of the electric field. Dashed lines indicate frequencies of observed whistler waves $\left(f_{0}\right.$ and $\left.f_{1}\right)$ and the frequency of EAW $\left(f_{0}-f_{1}\right)$. Crosses indicate the supposed positions of peaks on cross-frequencies $\left(\left[f_{0}, f_{1}\right]\right.$ and $\left.\left[f_{1}, f_{2}\right]\right)$ in a case of their phase connection.

waves of the sound type that evolve from sinusoidal waves into chains of sawtooth structures [Whitham, 1974]. To understand the evolution of EAW into TDS, it is first noted that, because the velocities of the hot electrons are larger than the phase velocity of the waves, they are distributed in the field of the electrostatic potential $\varphi$ of the wave according to

$$
n_{\mathrm{eH}}=n_{\mathrm{eH} 0} \exp \left(\frac{e \varphi}{k_{B} T_{\mathrm{eH}}}\right) .
$$

This dependence signifies that the density of the hot electron population is determined by the potential, but it also shows that the electric field is uniquely related to the gradient of this density of these electrons:

$$
E_{z}=-\frac{k_{B} T_{\mathrm{eH}}}{e} \frac{1}{n_{\mathrm{eH}}} \frac{\partial n_{\mathrm{eH}}}{\partial z} .
$$

This equation sheds light on the evolution of the wave electric field from the quasi-sinusoidal wave to electric field bursts. EAWs are compressible perturbations and, in the long wavelength limit, these perturbations evolve from the sinusoidal density perturbations to chains of sawtooth type perturbations of density. It is well known from classical gas dynamics that the sawtooth density and velocity profiles correspond to jumps or bursts and to the formation of gradient catastrophes of the spatial derivative of the density followed by density discontinuities [Whitham, 1974]. The relationship of the electric field of the wave to the derivative in the EAW leads to the conclusion that the electric field bursts are the result of steepening of the density profile that is the major feature of the dynamics of compressible wave perturbations of the EAW type. From the EFW measurements aboard the Van Allen Probes spacecraft it follows that the characteristic scale of the bursts is about $0.3-1 \mathrm{~km}$, corresponding to several $\lambda_{\mathrm{eH}}$, as it is supposed to be.

Additionally, the indication of the interaction between the observed waves can be obtained from their phase relationship by use of the wavelet bicoherence technique [Dudok de Wit and Krasnosel'skikh, 1995; Van Milligen et al., 1995]. The phase, $\phi\left(\omega_{0}\right)$ of wave $\exp \left[-i \omega_{0} t+i \phi\left(\omega_{0}\right)\right]$, is related to the phase, $\phi\left(\omega_{1}\right)$ of the second wave as

$$
\phi\left(\omega_{0}\right)+\phi\left(\omega_{1}\right)-\phi\left(\omega_{0}-\omega_{1}\right)=\text { constant }
$$


for the three-wave parametric decay that satisfies $\omega_{0}-\omega_{1}=\omega_{2}$. The bicoherence is a measure of this phase correlation. It is equal to 1 at frequency pairs that satisfy the above equation and is 0 in the presence of randomly phased Gaussian noise [Kravtchenko-Berejnoi et al., 1994]. Thus, in a real situation in which there is a mixture of random noise and parametric decay, the bicoherence would be between 0 and 1 . The wavelet bicoherence measured from $B_{x}, B_{y}$, and $E_{z}$ is shown in Figure 3. The peak with amplitude $\sim 0.3$ is near the frequency pair of $1500 \mathrm{~Hz}$ and $1800 \mathrm{~Hz}$ (indicated by crosses in the plot), which corresponds well with the two observed whistler waves. This result supports the interpretation that the lower frequency whistler and the low-frequency TDS resulted from the parametric decay of the higher-frequency whistler wave.

\section{Discussion and Conclusions}

In this paper we investigate the parametric resonant interaction of three waves: two VLF whistler waves (with frequencies $\omega_{0}$ and $\omega_{1}$ ) propagating in opposite directions and low-frequency electron acoustic wave (frequency $\omega_{2}$ roughly being the difference between the whistler wave frequencies) which transforms to the nonlinear localized bursts of electric field with the main component parallel to the background magnetic field. The Van Allen Probes spacecraft often observes clusters of such spatially localized bursts in the Earth's outer radiation belts, and often they are associated with large-amplitude chorus waves. These observed structures propagate to the geomagnetic equator and share properties of soliton-like nonlinear electron acoustic waves: a velocity of propagation $\sim 2000-4000 \mathrm{~km} / \mathrm{s}$ and a spatial scale of electric field burst periodicities $\sim 20-40 \mathrm{~km}$. Observed properties of the three waves are in a good agreement with an assumption of their parametric interaction. The bicoherence analysis indicates phase relation of the electron-acoustic wave with the two whistler waves. This mechanism of induced parametric interaction can produce quasiperiodic TDS in the outer Van Allen radiation belt.

\section{Acknowledgments}

The work by O.A., F.M., and V.K. was performed under JHU/APL contract 922613 (RBSP-EFW). The work of A.V.A was partially supported by MK-1781.2014.2. We thank Didier Mourenas for the fruitful discussions. We also thank all the people associated with the electric field, magnetic field, the particle teams on the Van Allen Probes, and the project team at the Johns Hopkins Applied Physics Laboratory that manages this program. All data used in this paper can be found at RBSP/EFW database (http://www.space.umn.edu/missions/ rbspefw-home-university-of-minnesota/).

The Editor thanks two anonymous reviewers for their assistance in evaluating this paper.

\section{References}

Agapitov, O., A. Artemyev, V. Krasnoselskikh, Y. V. Khotyaintsev, D. Mourenas, H. Breuillard, M. Balikhin, and G. Rolland (2013a), Statistics of whistler mode waves in the outer radiation belt: Cluster STAFF-SA measurements, J. Geophys. Res. Space Physics, 118, 3407-3420, doi:10.1002/jgra.50312.

Agapitov, O., V. Krasnoselskikh, Y. V. Khotyaintsev, and G. Rolland (2013b), Correction to A statistical study of the propagation characteristics of whistler waves observed by Cluster, Geophys. Res. Lett., 39, L24102, doi:10.1029/2012GL054320.

Artemyev, A. V., O. V. Agapitov, F. Mozer, and V. Krasnoselskikh (2014), Thermal electron acceleration by localized bursts of electric field in the radiation belts, Geophys. Res. Lett., 41, 5734-5739, doi:10.1002/2014GL061248.

Bernstein, I. B., J. M. Greene, and M. D. Kruskal (1957), Exact theory of nonlinear plasma oscillations, Phys. Rev., $108,546$.

Chen, L. (1977), Parametric excitation of 'kinetic' Alfven waves by whistler waves, Plasma Phys., 19, 47, doi:10.1088/0032-1028/19/1/006.

Drake, J. F., O. V. Agapitov, and F. S. Mozer (2015), The development of a bursty precipitation front with intense localized parallel electric fields driven by whistler waves, Geophys. Res. Lett., 42, doi:10.1002/2015GL063528.

Dudok de Wit, T., and V. V. Krasnosel'skikh (1995), Wavelet bicoherence analysis of strong plasma turbulence at the Earth's quasi-parallel bow shock, Phys. Plasmas, 2, 4307-4311.

Fried, B. D., and R. W. Gould (1961), Longitudinal ion oscillations in a hot plasma, Phys. Fluids, 4, 139.

Galeev, A. A., and R. Z. Sagdeev (1973), Nonlinear Plasma Theory, Reviews of Plasma Physics, vol. 7, edited by M. A. Leontovich, p. 3, Consultants Bureau, New York.

Helliwell, R. A. (1965), Whistlers and Related lonospheric Phenomena, Stanford Univ. Press, Stanford, Calif.

Holloway, J. P., and J. J. Dorning (1991), Undamped plasma waves, Phys. Rev. A, 44, 3856-3868.

Kletzing, C. A., et al. (2013), The Electric and Magnetic Field Instrument Suite and Integrated Science (EMFISIS) on RBSP, Space Sci. Rev., 179, 127-181, doi:10.1007/s11214-013-9993-6.

Kravtchenko-Berejnoi, V., V. Krasnoselskikh, D. Mourenas, and F. Lefeuvre (1994), Higher-order spectra and analysis of a non-linear dynamic model, Cluster Workshop on Data Analysis Tools, Braunschweig, Germany, ESA SP-371, p. 61.

Malaspina, D. M., L. Andersson, R. E. Ergun, J. R. Wygant, J. W. Bonnell, C. Kletzing, G. D. Reeves, R. M. Skoug, and B. A. Larsen (2014), Nonlinear electric field structures in the inner magnetosphere, Geophys. Res. Lett., 41, 5693-5701, doi:10.1002/2014GL061109.

Montgomery, D. S., R. J. Focia, H. A. Rose, D. A. Russell, J. A. Cobble, J. C. Fernandez, and R. P. Johnson (2001), Observation of stimulated electron-acoustic-wave scattering, Phys. Rev. Lett., 87, 155001.

Mozer, F. S., S. D. Bale, J. W. Bonnell, C. C. Chaston, I. Roth, and J. Wygant (2013), Megavolt parallel potentials arising from double-layer streams in the Earth's outer radiation belt, Phys. Rev. Lett., 111(23), 235002, doi:10.1103/PhysRevLett.111.235002.

Mozer, F. S., O. Agapitov, V. Krasnoselskikh, S. Lejosne, G. D. Reeves, and I. Roth (2014), Direct observation of radiation belt electron acceleration from electron volt energies to megavolts by non-linear whistlers, Phys. Rev. Lett., 113, 035001.

Nikolic, Lj., M. M. Skoric, S. Ishiguro, and T. Sato (2002), Stimulated electron-acoustic-wave scattering in a laser plasma, Phys. Rev. E, 66, 036404.

Sato, T., and H. Okuda (1981), Numerical simulations on ion acoustic double layers, J. Geophys. Res., 86(A5), 3357-3368, doi:10.1029/JA086iA05p03357.

Schamel, H. (2000), Hole equilibria in Vlasov-Poisson systems: A challenge to wave theories of ideal plasmas, Phys. Plasmas, 7, 4831.

Stix, T. H. (1962), The Theory of Plasma Waves, p. 218, McGraw-Hill, New York.

Sircombe, N. J., T. D. Arber, and R. O. Dendy (2006), Aspects of electron acoustic wave physics in laser backscatter from plasmas, Plasma Phys. Controlled Fusion, 48, 1141 -1154, doi:10.1088/0741-3335/48/8/007.

Shukla, P. K. (1975), Parametric coupling of a large-amplitude whistler wave with ion quasimodes in plasmas, Phys. Rev. A, 16, 1294. 
Thorne, R. M. (2010), Radiation belt dynamics: The importance of wave particle interactions, Geophys. Res. Lett., 37, L22107, doi:10.1029/2010GL044990.

Van Milligen, B. Ph., E. Sanchez, T. Estrada, C. Hidalgo, B. Branas, B. Carreras, and L. Garcia (1995), Wavelet bicoherence: A new turbulence analysis tool, Phys. Plasmas, 2(8), 3017.

Vasko, I. Y., O. V. Agapitov, F. Mozer, A. V. Artemyev, and D. Jovanovic (2015), Magnetic field depression within electron holes, Geophys. Res. Lett., 42, 2123-2129, doi:10.1002/2015GL063370.

Whitham, G. B. (1974), Linear and Nonlinear Waves, John Wiley, New York.

Wygant, J. R., et al. (2013), The electric field and waves instruments on the radiation belt storm probes mission, Space Sci. Rev., 179, 183-220, doi:10.1007/s11214-013-0013-7. 